\title{
Contactless Healthcare
}

\section{Jong-Yeup Kim}

Department of Biomedical Informatics, Konyang University, College of Medicine, Daejeon, Korea

As coronavirus disease 2019 (COVID-19) is wreaking havoc across the world, and because of its strong propagation power, remote alternatives are being explored in many industries, including healthcare [1]. Various attempts have been made, including telemedicine, the remote monitoring of patients and chronic disease management, which are being launched as new services $[2,3]$. Thus, we aim to explore the trends and related issues of various contactless healthcare services.

In fact, among contactless healthcare services, telemedicine is already seamlessly operating in the United States, Japan, and China. Nevertheless, many countries, including the Republic of Korea, which has a relatively high rate of introduction of the hospital information system, have yet to even begin. Therefore, it is appropriate to view this as an issue occurring at the social consensus stage rather than a technical one. However, since in-person care cannot be prioritized in pandemics such as COVID-19, it is necessary to begin considering and discussing a number of issues related to telemedicine.

First, we must consider the issue of keeping medical records. The medical team will keep the records of the medical acts performed through telemedicine, which is the foundation for the continuity of care of patients during follow-up visits (next treatment appointment). However, for medical records to function properly, other medical staff must be able to consult them. Considering that the exchange of medical information is a critical topic in the era of Electronic Medical Records, we should preemptively consider the standards

This is an Open Access article distributed under the terms of the Creative Commons Attribution Non-Commercial License (http://creativecommons.org/licenses/by$\mathrm{nc} / 4.0 /$ ) which permits unrestricted non-commercial use, distribution, and reproduction in any medium, provided the original work is properly cited.

(C) 2020 The Korean Society of Medical Informatics to be set for the various telemedicine record formats [4].

Second, an agreement on the fees for telemedicine services is also critical. Some believe that telemedicine should be less expensive than face-to-face care. However, the time cost is the same for conventional healthcare and telemedicine, and telemedicine requires that additional devices be prepared. Therefore, nearly all doctors argued that it is reasonable to assume that telemedicine is more expensive than face-toface care. Evidently, the implementation of telemedicine will save socioeconomic costs, as the patient's traveling time to medical institutions will be saved and transportation costs will be nullified. Thus, it seems reasonable that the cost of implementing telemedicine services is partially compensated by the extent of the patient's cost reduction.

Third, discussions on telemedicine-related accidents are also needed. Doctors cite the possibility of a medical malpractice due to inaccurate diagnosis as the prime rebuttal against telemedicine. Diagnosing via telemedicine may be less accurate than a doctor's examination using all senses in face-to-face care. Therefore, the consensus seems appropriate in Japan, where telemedicine is performed within the scope of medical institutions that can be visited in person. In Japan, as further medical examinations can be required in addition to telemedicine, only medical institutions that can be promptly visited are authorized to conduct telemedicine [5].

Fourth, the essential problem with telemedicine is the delivery of medicines. The obligation for a patient who has been treated at home through telemedicine to leave the house to buy medicine renders telemedicine imperfect. Globally, the law states that professional medicine implies visiting pharmacies and receiving medication instructions from pharmacists, which is critical to prevent drug misuse. Therefore, rather than allowing or banning the delivery of all 
drugs, it is necessary to specify whether the drug is available for delivery by subdividing the ethical-the-counter (ETC) drug.

In addition, issues related to the remote monitoring of patients should be considered in contactless healthcare. For the growth of the remote monitoring industry, three predominant aspects must be considered.

The first is the development of new sensor devices. To monitor the condition of chronic patients, a large battery use capacity must be secured, the devices should be easy to calibrate, and reliable in their sensing value [6,7].

The second is the establishment of treatment guidelines using sensing data. Doctors still lack treatment experience based on the analysis of continuously monitored data. Therefore, clinical research should be conducted on treatment options according to monitoring data, and treatment guidelines should be established based on the findings [8].

Third, issues remain regarding the calculation of remote monitoring fees, and who will cover them. Since medical staff's time and effort is inevitable in remote monitoring and therapeutic interventions, there will be no discussion on the need for these medical acts to be charged. However, many variables must be considered when determining the fees. If the patient's chronic disease management is facilitated through remote monitoring and can effectively prevent complications, this could support arguments for saved social overhead costs.

Given the complexity of the needed COVID-19 response, I think that a return to normal life will be strenuous. Thus, we should accelerate discussions on various issues related to contactless healthcare to better adapt to the new normal.

\section{ORCID}

Jong-Yeup Kim (https://orcid.org/0000-0003-1230-9307)

\section{References}

1. Johnson C, Taff K, Lee BR, Montalbano A. The rapid increase in telemedicine visits during COVID-19. Patient Exp J 2020;7(2):72-9.

2. Volterrani M, Sposato B. Remote monitoring and telemedicine. Eur Heart J Suppl 2019;21(Suppl M):M54M56

3. Kim SY, Park JE, Seo HJ, Seo HS, Sohn HJ, Shin CM, et al. NECA's guidance for undertaking systematic reviews and meta-analyses for intervention. Seoul, Korea: National Evidence-based Healthcare Collaborating Agency; 2011.

4. Lenert L, McSwain BY. Balancing health privacy, health information exchange, and research in the context of the COVID-19 pandemic. J Am Med Inform Assoc 2020;27(6):963-66.

5. Oh JY, Park YT, Jo EC, Kim SM. Current status and progress of telemedicine in Korea and other countries. Healthc Inform Res 2015;21(4):239-43.

6. Hui TK, Sherratt RS, Sanchez DD. Major requirements for building Smart Homes in Smart Cities based on Internet of Things technologies. Future Gener Comput Syst 2017;76:358-69.

7. Chakraborty C, Gupta B, Ghosh SK. Mobile telemedicine systems for remote patient's chronic wound monitoring. In: Virtual and mobile healthcare: breakthroughs in research and practice. Hershey (PA): IGI Global; 2020. p. $977-1003$.

8. Liao Y, Thompson C, Peterson S, Mandrola J, Beg MS. The future of wearable technologies and remote monitoring in health care. Am Soc Clin Oncol Educ Book 2019;39:115-21. 